\title{
NUMERICAL IMPLEMENTATION OF THE COARSE-STEP METHOD WITH A VARYING DIFFERENTIAL-GROUP DELAY
}

\author{
M. Eberhard ${ }^{1}$ and C Braimiotis ${ }^{1}$ \\ ${ }^{1}$ Photonics research group, Engineering department, Aston university, Birmingham, U.K
}

\begin{abstract}
The effect of having a fixed differential-group delay term in the coarse-step method results in a periodic pattern in the autocorrelation function. We solve this problem by inserting a varying DGD term at each integration step, according to a Gaussian distribution. Simulation results are given to illustrate the phenomenon and provide some evidence, about its statistical nature.
\end{abstract}

\section{INTRODUCTION}

As demonstrated in [2] the autocorrelation function (ACF) produced after the use of the coarse-step method, deviates from the analytical model. A repetitive pattern appears, owing to the additive effect of the convolution, in the time domain of the signal with a fixed differential group delay (DGD) term, at each integration step. Moreover it is possible, to minimise this effect by allowing the DGD coefficient to change at each step as a Gaussian variate.

\section{REVIEW OF THE COARSE-STEP METHOD}

Following in the derivation of [1] the starting point is the coupled nonlinear Schroedinger equation (CNLS),

$i \frac{\partial \Psi}{\partial z}+\tilde{\Sigma} \Psi+i b^{\prime} \sigma_{3} \frac{\partial \Psi}{\partial t}-\frac{1}{2} \beta^{\prime \prime} \frac{\partial^{2} \Psi}{\partial t^{2}}+n_{2} k_{0}\left[\frac{5}{6}|\Psi|^{2} \Psi+\frac{1}{6}\left(\Psi^{\dagger} \sigma_{3} \Psi\right) \sigma_{3} \Psi+\frac{1}{3} N\right]=0$

Should be denoted that, $\Psi=R(z) A$ where $R(z)$ and $\tilde{\Sigma}$ are the following matrices, 


$$
\begin{gathered}
R(z)=\left(\begin{array}{cc}
\cos \alpha & \sin \alpha \\
-\sin \alpha & \cos \alpha
\end{array}\right) \\
\tilde{\Sigma}=\left(\begin{array}{cc}
b & -i \alpha_{z} \\
i \alpha_{z} & -b
\end{array}\right)
\end{gathered}
$$

Equations 2 and 3 follow the rapid evolution of the field along distance $\mathrm{z}$, as the direction of the birefringence axes will be rapidly changing. Where the birefringence parameter $b=\left(\beta_{1}-\beta_{2}\right) / 2$ and the specific group delay per unit length $b^{\prime}=\left(\beta_{1}^{\prime}-\beta_{2}^{\prime}\right) / 2$. In the CNLS $n_{2}$ is the Kerr coefficient while $k_{0}=\frac{2 \pi}{\lambda}$ is the wavenumber. As indicated in [1] if the birefringence axes are fixed then the angle of rotation equals zero, $\alpha_{z}=d \alpha_{z} / d z=0$ and thus $N=\left(\Psi_{1}^{*} \Psi_{2}^{2}, \Psi_{1}^{2} \Psi_{2}^{*}\right)^{t}$ varies rapidly and can be dropped as observed in $[3,4]$. Equation 1 then becomes,

$$
i \frac{\partial \Psi}{\partial z}+i b^{\prime} \sigma_{3} \frac{\partial \Psi}{\partial t}-\frac{1}{2} \beta^{\prime \prime} \frac{\partial^{2} \Psi}{\partial t^{2}}+n_{2} k_{0}\left[\frac{5}{6}|\Psi|^{2} \Psi+\frac{1}{6}\left(\Psi^{\dagger} \sigma_{3} \Psi\right) \sigma_{3} \Psi\right]=0
$$

When $b^{\prime} \neq 0$ the signal is subjected to polarization mode dispersion(PMD), while the third term causes chromatic dispersion.

Assuming that the step size is large enough so that the field has lost memory of its initial polarization, the solution of the CNLS can be multiplied by a scattering matrix $\mathrm{S}$ so that the polarization is randomly reorientated.

$$
S=\left(\begin{array}{cc}
\cos \alpha & \sin \alpha \exp (i \phi) \\
-\sin \alpha \exp (i \phi) & \cos \alpha
\end{array}\right)
$$

The multiplication of the signal at different frequencies with a fixed DGD term induces a periodicity in the autocorrelation function as it is shown through our results. The ACF thus deviates from the theoretical model. This effect is eliminated including a varying DGD from step-to-step and consequently averaging out the unwanted peaks that are present in the ACF.

\section{AUTOCORRELATION FUNCTION}

The simulations performed on a system having the following characteristics $D_{P M D}=3 \mathrm{ps} / \mathrm{sqrt}(\mathrm{km})$, correlation length $L_{c}=100 \mathrm{~m}$, integration step 1 $\mathrm{km}$ while the optical bandwidth of the simulation is $4 \mathrm{THz}$. The ACF was compared with the following function $[5,6]$, where $\Delta \tau$ is the mean DGD,

$$
A C F_{\text {analytical }}=\frac{3}{\Delta \tau^{2}\left(\omega-\omega_{0}\right)^{2}}\left[1-\exp \left(\frac{-\Delta \tau^{2}\left(\omega-\omega_{0}\right)^{2}}{3}\right)\right]
$$


and calculated from the simulations according to the following formula,

$$
A C F_{\text {analytical }}=\left|\frac{\langle\Omega(\omega)\rangle\left\langle\Omega\left(\omega_{0}\right)\right\rangle}{\left\langle\Omega\left(\omega_{0}\right)\right\rangle\left\langle\Omega\left(\omega_{0}\right)\right\rangle}\right|
$$

$\Omega(\omega)$ is the polarization dispersion vector calculated as in [2]. As can be shown in figure 1, using a fixed value DGD we are getting the periodic pattern in the ACF of the coarse-step method.

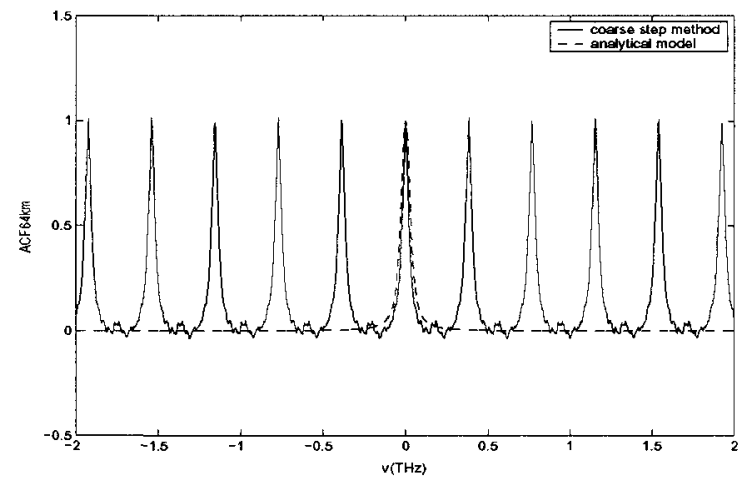

Figure 1. ACF of coarse step method with a fixed DGD of $3 \mathrm{ps}$ for $64 \mathrm{~km}$.

Allowing the DGD to vary as a random Gaussian variate with a standard deviation $\sigma$ ranging from $0.009-1 \mathrm{ps}$ minimizes the problem.

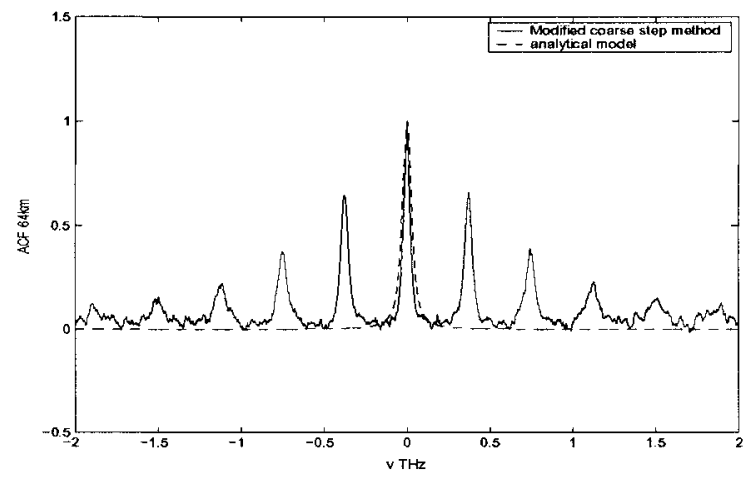

Figure 2. ACF of coarse step method with DGD varying according to a Gaussian distribution of $\sigma=0.09 \mathrm{ps}$ and mean $\mu=3 p s$ for $64 \mathrm{~km}$.

It is obtained through the simulation results that the harmonics of the ACF gradually diminish as the standard deviation of the distribution is getting larger. 


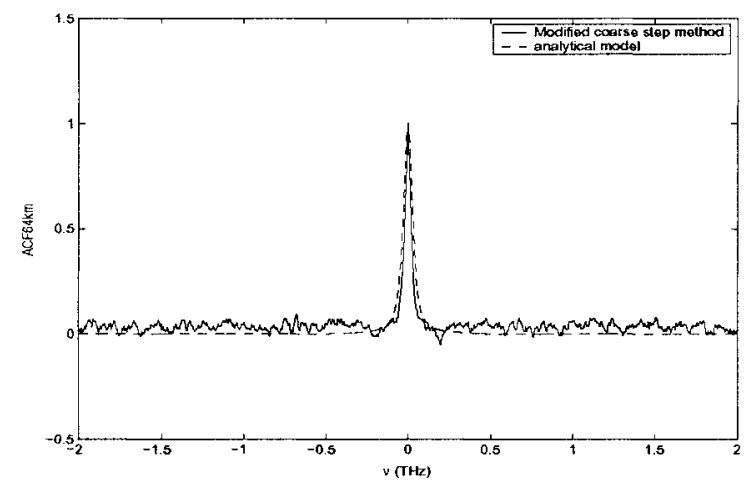

Figure 3. ACF of coarse step method with DGD varying according to a Gaussian distribution of $\sigma=1 p s$ and mean $\mu=3 p s$ for $64 \mathrm{~km}$.

It is worth noting that the PDF of the DGD does not change when we modify the coarse-step method, as it is shown in figure 4.

Moreover we can follow the evolution of $\sigma$, through our simulations by following the difference $\Delta \mathrm{A}$ between the centre peak amplitude and the first harmonic amplitude as presented in figure 5. From the graph we can didact that at a value of $\sigma=0.6 \mathrm{ps}$, the peak of the first harmonic drops at 10 percent, of its original value.

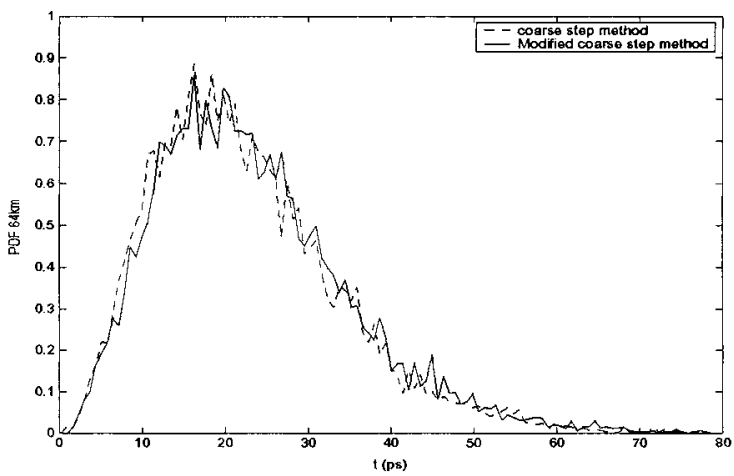

Figure 4. comparison of the PDF of the coarse-step method with a fixed DGD with a modified coarse-step method using a DGD varying according to a Gaussian distribution of $\sigma=0.09 p \mathrm{~s}$ and mean $\mu=3 p s$ for $64 \mathrm{~km}$. 


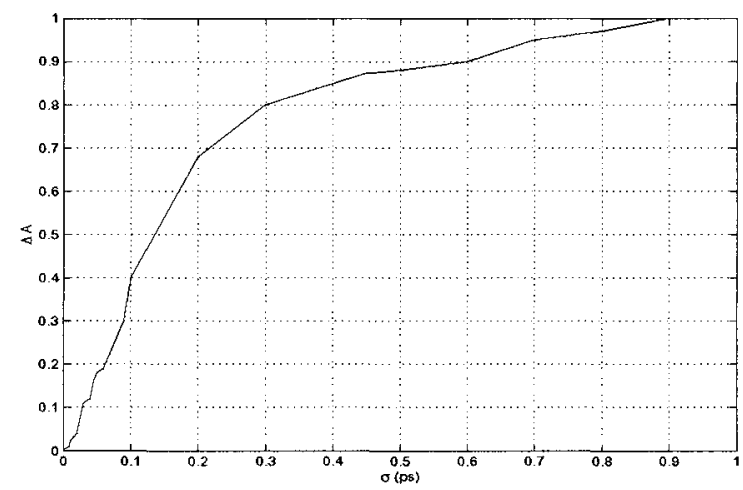

Figure 5. Evolution of the difference in amplitude between the fundamental frequency and the first harmonic for different values of $\sigma$.

\section{CONCLUSION}

Instead of using a fixed DGD we inserted a Gaussian variate, so that the peaks of ACF of the coarse-step method average out and the numerical implementation outcome agrees with the theoretical results. Moreover we provided simulation results that illustrate the decrease in the amplitude of the side peaks of ACF and give some evidence about a future analytical treatment of the phenomenon.

\section{REFERENCES}

[1] D. Marcuse, C.R. Menyuk and P.K.A. Wai, "Application of the Manakov-PMD equation to studies of signal propagation in optical fibers with randomly varying birefringence", J.Lightwave Tech. 15, 1735-1746(1997).

[2] M. Eberhard and C. Braimiotis, "Numerical implementation of the Manakov-PMD equation with precomputed $M(\omega)$ matrices", Nonlinear Guided Waves and Their Applications proceedings,MC3(2004).

[3] C.R. Menyuk, "Nonlinear pulse propagation in birefringence optical fibers", IEEE J.Quantum Electron., vol. 23, pp. 174 - 176(1987).

[4] C.R. Menyuk, "Stability of soltons in birefringent optical fibers. I: Equal propagation amplitudes", Opt. Lett., vol. 12, pp. 614-616, 1987; see also, C.R.Menyuk,"Stability of solitons in birefringent optical fibers.II:Arbitrary amplitudes" J. Opt. Soc. Amer. B., vol. 5, pp.392 -402(1988).

[5] M. Shtaif, A. Mecozzi and J.A.Nagel, "Mean-square magnitude of all-order polarization mode dispersion and the relation with the bandwidth of proncipal states", IEEE Photon. Tech. Lett.12, 53-55(2000).

[6] M. Karlsson and J. Brentel, "Autocorrelation function of the polarization mode dispersion vector", Opt. Lett. 24, 497-469(1999). 